\title{
Improving Still Image Coding by an SOM-Controlled Associative Memory
}

\author{
Gerald Krell $^{1}$, René Rebmann ${ }^{1}$, Udo Seiffert ${ }^{2}$, and Bernd Michaelis ${ }^{1}$ \\ 1 Otto-von-Guericke University Magdeburg, Germany, \\ krell@iesk.et.uni-magdeburg.de, \\ http://iesk.et.uni-magdeburg.de/ ${ }^{\mathrm{krell}}$ \\ ${ }^{2}$ Leibniz Institute of Plant Genetics and Crop Plant Research Gatersleben, Germany
}

\begin{abstract}
Archiving of image data often requires a suitable data reduction to minimise the memory requirements. However, these compression procedures entail compression artefacts, which make machine processing of the captured documents more difficult and reduce subjective image quality for the human viewer. A method is presented which can reduce the occurring compression artefacts. The corrected image yields as output of an auto-associative memory that is controlled by a Self-Organising Map (SOM).
\end{abstract}

\section{Introduction}

Standard image compression algorithms do not consider the image content for the selection of compression parameters. Often, the user has to do experiments with the compression parameters until his requirements are met. We propose a system for the correction of compression artefacts based on an associative memory that is improved by a Self-Organizing Map (SOM) controlling its parameters (Fig. 1).

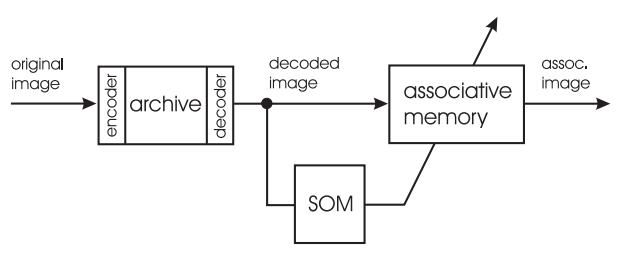

Fig. 1. SOM-controlled associative memory

The JPEG procedure (Joint Photographic Expert Group) [2]3 is typical for lossy image compression and an inherent part of image capturing devices such as scanners or digital cameras often implemented in integrated circuits. The classical block-based JPEG procedure formed therefore the basis of our fundamental investigations, but the main ideas of our approach can be applied also to the wavelet-based methods of JPEG 2000. The examples are on the basis 
of gray-value images in order to simplify the explanations. The same method can be applied to each component of color images, of course. Consideration of the coupling of the basis colors can improve the results but is not further explained in this paper.

Different approaches exist to describe image degradation [11. Often, the deviation of the considered and the error-free reference image is assessed by the sum or mean of error squares (or the square root of it: root mean square, RMS) as a quantitative measure. Also the signal-to-noise ratio (SNR) and the mean absolute difference (MAD) are important criterions. The integrating property of these criteria is a serious drawback and the subjective evaluation of an image often deviates from such simple measures. Subjective assessment of image quality depends strongly on the assessing group of persons [11]. In order to reduce the compression artefacts generated by JPEG compression, different solutions have been examined. The quantitative error measure for all investigated approaches has been the sum of error squares. This avoids expensive interviews for a subjective evaluation and the results are easier to be compared. In most cases, a lower quadratic error leads to a better subjective image quality.

An important assumption in our approach is that images of a certain image content can be assigned to classes of images. The variability of an image is limited in many typical cases such as text documents, cheque forms, traffic scenes, nature images etc. Internal coherences exist in the images of an image class, which should be generally exploited for redundancy reduction. This way, the compression procedure is optimised depending on the particular image class. The application of a procedure with well defined parameters for a particular image class to a different (dissimilar) image class can therefore lead to poor results. An image-class-dependent correction seems to be a promising approach to compensate the loss of information caused by image compression. The missing information on the coherences within an image class is recalled from an associative memory after the decompression. This way, the compression artefacts are to be reduced.

\section{An Auto-associative Recall for Compressed Image Blocks}

At first we describe the function of the associative memory. It is applied to the degraded archive images of a certain class. To consider calculation resources and the typical structure of JPEG we have designed associative memories according to image blocks of 8 by 8 pixels. For the $k$-th block of an image yields

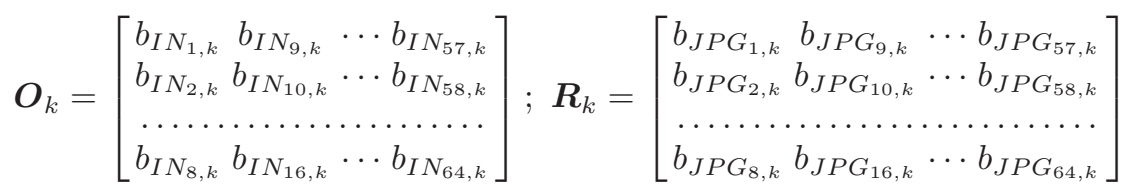

where $\boldsymbol{O}_{k}$ is the original image block and $\boldsymbol{R}_{k}$ the resulting block after compression/decompression with $b_{I N_{1 \ldots 64, k}}$ and $b_{J P G_{1 \ldots 64, k}}$ being the $8 * 8$ gray values of 
the $k$-th image blocks. The gray values are numbered column-wise obtaining the vectorized original and compressed/decompressed images, respectively:

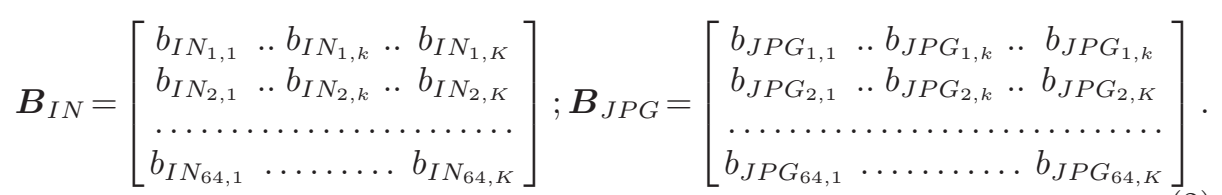

These $K$ blocks can originate from the same image or from different images but their contents should possess similar properties to fulfil the desired requirement to represent a certain image class. The selection of suitable sample blocks presenting prototypes of the defined image class is crucial for a good correction. Different strategies to determine the sample blocks have been therefore investigated. For the description of the associative memory approach, we will now use Fig. 2.

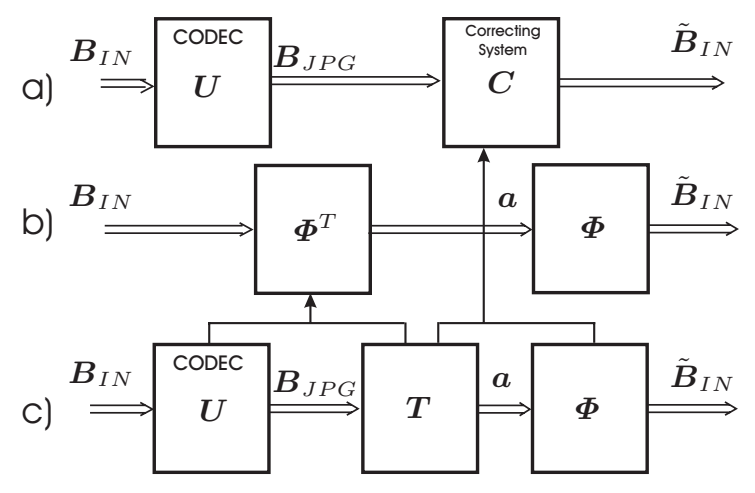

Fig. 2. Associative memory for the correction of compression artefacts

Investigations on real image data showed that the relationships between an original and a decompressed image can be approximately described by a set of linear equations. This holds, of course, especially for image classes that are characterized by certain statistic properties. The process of compression/decompression (CODEC) can therefore be approximated by a system matrix $\boldsymbol{U}$ for a mathematically simple description (see Fig. 2a). Assuming an approximately linear transfer behaviour of the CODEC, the elements of $\boldsymbol{U}$ can be determined by minimisation of the error squares for a test data set. The number of samples must be greater than the number of pixels in the image block. This corresponds to the determination of the pseudo inverse [84]:

$$
\boldsymbol{U}=\boldsymbol{B}_{J P G} \boldsymbol{B}_{I N}^{T}\left(\boldsymbol{B}_{I N} \boldsymbol{B}_{I N}^{T}\right)^{-1} .
$$

We are looking for a correcting system $\boldsymbol{C}$ that compensates the influences caused by $\boldsymbol{U}$ as far as possible. This means that our aim is to approximate $\tilde{\boldsymbol{B}}_{I N}$ 
by $\boldsymbol{B}_{I N}$. Such systems can be considered as auto-associative memories. The investigation of the eigenvalues of the transfer matrix $\boldsymbol{U}$ resulted in a certain number of eigenvalues with a value zero or close to zero. Here, a direct relationship to the lossy data compression exists where higher frequency components are also often weighted less (reduced code length of JPEG for coefficients of higher frequency components). More eigenvalues of $\boldsymbol{U}$ are close to zero with increasing compression rate (lower quality). The relation between number of zero values at higher frequencies and the compression rate is almost linear. This observed behaviour motivates a the scheme of classical auto-associative memory [1] according to Fig. 2b that is abel to store a-priori knowledge on the image contents. The determination of $\boldsymbol{\Phi}$ for a certain data set can be considered as a training that approximates $\tilde{\boldsymbol{B}}_{I N}$ by $\boldsymbol{B}_{I N}$ (auto-association). The training of the auto-associative system can be expressed by

$$
\tilde{\boldsymbol{B}}_{I N}=\boldsymbol{\Phi} \boldsymbol{\Phi}^{T} \boldsymbol{B}_{I N} \rightarrow \boldsymbol{B}_{I N}
$$

with a residual error caused by the interpolating behaviour and

$$
\tilde{B}_{I N}=\sum_{\nu} a_{\nu} \varphi_{\nu}=\boldsymbol{\Phi} \boldsymbol{a}
$$

with $\boldsymbol{\varphi}_{\nu}$ being the $\nu$-th line of $\boldsymbol{\Phi}$ and $a_{\nu}$ the $\nu$-th dimension-reduced signal. The mean square error between $\tilde{\boldsymbol{B}}_{I N}$ and $\boldsymbol{B}_{I N}$ has to be minimized for each length of vector $\boldsymbol{a}$. An eigenvalue problem results when considering the data of an image class (assuming the number of samples in the data set of this image class is much greater than one) as follows:

$$
\boldsymbol{B}_{I N} \boldsymbol{B}_{I N}^{T} \boldsymbol{\Phi}=\boldsymbol{\Lambda \Phi} .
$$

The transformation matrix $\boldsymbol{\Phi}$ is calculated by the solution of the eigenvalue problem [46]. The matrix $\boldsymbol{\Lambda}$ contains the eigenvalues of $\boldsymbol{B}_{I N} \boldsymbol{B}_{I N}{ }^{T}$ in the main diagonal. This dimension reduction is crucial for the overall system because it stands for the inherent a-priori knowledge that is stored in $\boldsymbol{\Phi}$. The corrected image is recalled from the left part of the associative memory (b) by the Karhunen Loève coefficients $\boldsymbol{a}$.

Because the CODEC produces image data a transfer matrix $\boldsymbol{T}$ producing vector $\boldsymbol{a}$ (Fig. 2 b) is separated from $\boldsymbol{\Phi}^{T}$. The behaviour of the eigenvalues of $\boldsymbol{U}$ (increasing compression $\rightarrow$ fewer eigenvalues $\neq 0$ ) leads to the conclusion that the first part of the auto-associative memory (Fig. 2b,can be considered as forward transform) models the process of compression/decompression if the length of vector $\boldsymbol{a}$ is limited in (Fig. 2k). The same holds for the correcting system $\boldsymbol{C}$ consisting of $\boldsymbol{T}$ and $\boldsymbol{\Phi}$.

$\boldsymbol{B}_{J P G}=\boldsymbol{U} \boldsymbol{B}_{I N}$ models the compression/decompression approximation by the associative memory and from Fig. 2 c follows $\tilde{\boldsymbol{B}}_{I N}=\boldsymbol{\Phi} \boldsymbol{T} \boldsymbol{B}_{J P G}$. This leads to $\boldsymbol{T}=\boldsymbol{U}^{-1} \boldsymbol{\Phi}^{T}$, with $\boldsymbol{U}^{-1}$ being the Moore-Penrose inverse 4 and to the resulting correction matrix

$$
\boldsymbol{C}=\boldsymbol{T} \boldsymbol{\Phi}=\left(\boldsymbol{U}^{-1} \boldsymbol{\Phi}^{T}\right) \boldsymbol{\Phi}
$$




\section{An SOM for Image Block Classification}

A further improvement of the results of correction can be expected if special transformation matrices $\boldsymbol{\Phi}$ and $\boldsymbol{T}$ are determined according to each single image class. An automatic and adaptive classification is desirable. Artificial neural networks are well known and suitable for classification tasks.

Because a direct learning target for the image blocks and the centre of gravity of the classes is hard to define, a SOM seems to be appropriate for this application. The SOM was suggested and developed by Kohonen in the early ' 80 s [ 5 ] and was then established as powerful and adaptive tool for clustering and visualization [10]. It belongs to the group of unsupervised trained artificial neural networks with a close relationship to biological signal processing [7]. Due to its inherent properties and biological origin the SOM seems to be predestined to be implemented in the complex image processing system described in this paper.

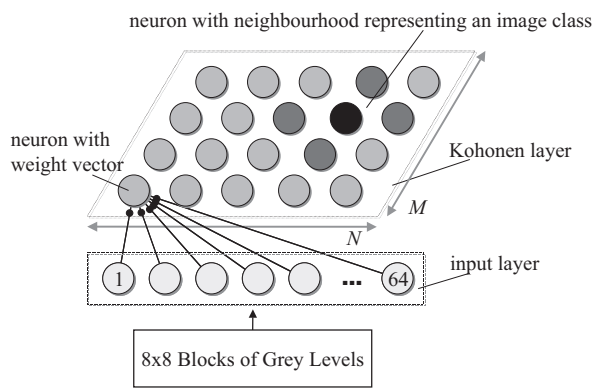

Fig. 3. Scheme of the SOM for the creation of image block classes

It performs an ordered non-linear mapping of high-dimensional input data onto a usually two-dimensional rectangular grid of neurons. This reduction of the dimensionality is characterised by an abstraction to important properties contained in the input data. At the same time insignificant information is reduced or even removed. The SOM generally allocates more neurons for inputs that occur more frequently in the input space (magnification factor). This improves the local resolution in these areas. This way the resources, in other words the usage of neurons available for the input data representation, are optimised during the training phase of the network. This is very similar to biological brain maps where for instance in the visual cortexa a larger cortical area is allocated for frequently presented observations.

Due to its topology preservation, the SOM, unlike many other clustering algorithms, keeps similarities of the input data by transforming these into neighbourhood relations of the organized map (Fig. 3). In this organzing phase each neuron matures to a prototype for a particular (sub-)cluster by adapting its own properties (weight vector) to those of a group of similar or identical input patterns (input vectors) by using a simple similarity criterion. A neighbourhood 


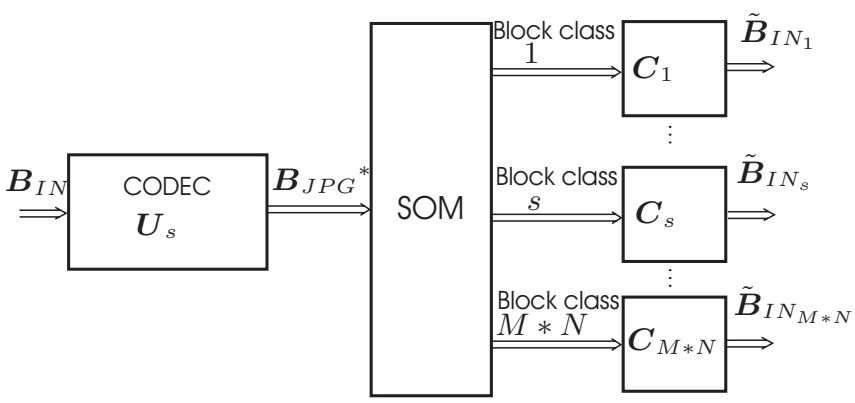

Fig. 4. SOM-controlled correction of the compression artefacts

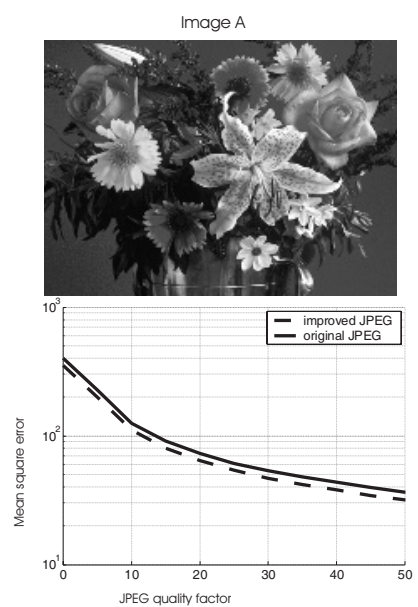

\section{Es sir transfor Betrach}



Image C
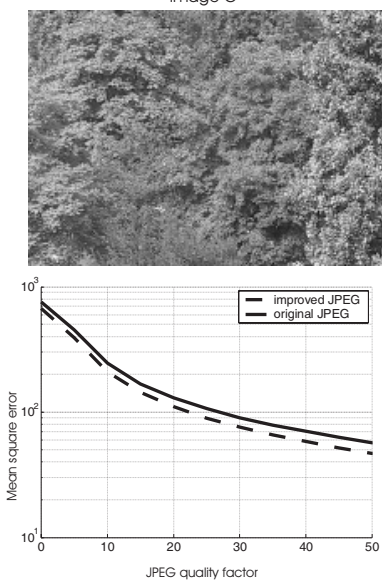

Fig. 5. Error reduction on the basis of example images without SOM

function works in such a way that neurons situated close to each other on the map are representing similar properties. In our case the input layer of the SOM is provided by the pixels of the image block. For each input one of the $M * N$ output neurons - the winner neuron - defines the represented image class. Fig. 4 shows the interaction of the SOM with the CODEC providing its input and the parameter sets $\boldsymbol{C}_{s}$ of the auto-associative memory to be activated by the winning neuron. For each block class, $1 \cdots s \cdots M * N$, a parameter set $\boldsymbol{\Phi}_{s}$ for the associative memory is calculated by solving the eigenvalue problems and similar to the former considerations (see Eq. 2) yields for each block class $s$ :

$$
\boldsymbol{C}_{s}=\left(\boldsymbol{U}_{s}^{-1} \boldsymbol{\Phi}_{s}^{T}\right) \boldsymbol{\Phi}_{s}
$$

\section{Results}

On the basis of three example images the results of the reduction of the compression errors (method of section 2, without SOM) are represented in the following 
Fig. 5 The compression error can be reduced by approximately $20 \%$ depending upon the image content and the quality factor or the compression rate can be approximately increased by $25 \%$ without rising up the compression error.

The proposed procedure for the reduction of the compression artefacts has positive effects on further automatic processing of the images. In order to obtain further improvements for the human viewer, other criteria must be additionally included in the selected error measure. The positive effect of reduced compression errors for further automatic processing can be demonstrated by the example of the text recognition. The recognition error can be reduced up to $48 \%$ at higher compression rates (quality factor less than 10). Typical image classes are classified by the SOM. Block-dependent image classes are obtained and the above described method can be applied with varying parameter sets to the associative memory.
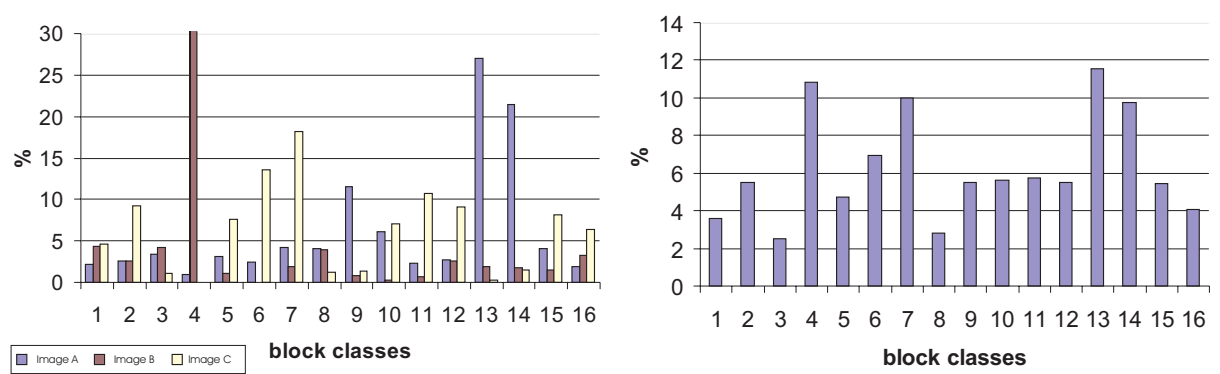

Fig. 6. Distribution of the image blocks after the training of a $4 \times 4$ SOM (left) for all blocks (right) ordered by sample images

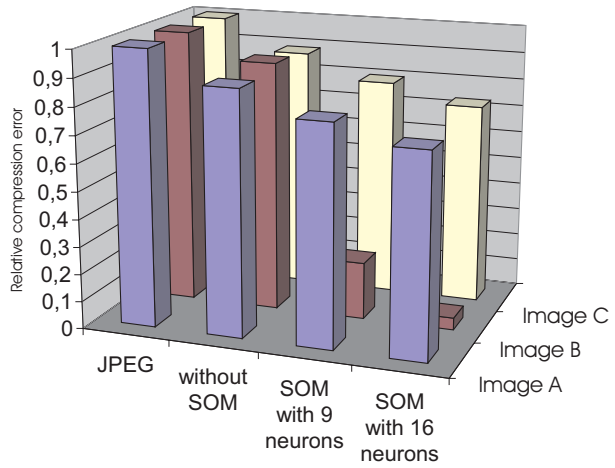

Fig. 7. Compression error without correction, with correction by associative memory but without SOM and correction error with associative memory controlled by SOM of different sizes, applied to the sample images 
Fig. 6 shows a training result of the SOM for the example images of Fig 4 The classifying neurons are well distributed. This is an indication th at the SOM is of reasonable size.

Fig. 7 shows the gain of quality by introducing the classifying SOM for the correcting system. With the varying parameter set for the associative memory, the error reduces by about $20 \cdots 30 \%$ for the gray tone images and up to 90 $\%$ for the text image. For simplification of the complex diagram, only relative compressions errors are indicated.

\section{Conclusion}

A system for compensation of compression errors by an associative memory has been presented. The performance has been increased considering the image (or block) content introducing a neural network based classification system - a SOM. The SOM enables us to apply the same correcting system to different types of image and also to images with strongly varying image content (for example mixture of text and photographs). The number of different parameter sets needed for correction is equal to the number of neurons in the SOM or the number of introduced image classes. [9] proposes an SOM as a compression scheme as an alternative to vector quantization directly generating the codewords. This interesting approach for compression with an SOM could also be completed with an SOM controlled associative memory as proposed in this paper. Depending on the codeword the associative memory is recalled with different parameter sets.

Acknowledgments. The investigations took place in the context of the project "Adaptive Read". The project is sponsored by the Federal Ministry for Education and Research of the Federal Republic of Germany (01 IN $902 \mathrm{~J}$ ) and by the federal state Sachsen-Anhalt (0032KB099).

\section{References}

1. Abbas H.M., Fahmy, M.M.: A Neural Model for Adaptive Karhunen Loéve Transformation (KLT).Proc. International Joint Conference on Neural Networks, Baltimore, Maryland, vol. 2 (1992)975-980

2. ISO/IEC JTC1 10918-1, ITU-T Rec. T.81, Information technology - Digital compression and coding of continuous-tone still images: Requirements and guidelines (1994)

3. ISO/IEC JTC 1/SC 29/WG 1: Coding of Still Pictures - JPEG 2000. Part 1 Final Committee Draft Version 1.0 (2000)

4. A. Kielbasinski; H. Schwetlick: Numerische Lineare Algebra. Deutscher Verlag der Wissenschaften, Berlin (1988)

5. Kohonen, T.: Self-Organizing Maps. 3rd ed., Springer, London (2001)

6. T. Lilienblum, P. Albrecht, R. Calow, B. Michaelis: Dent Detection in Car Bodies. 15th International Conference on Pattern Recognition (ICPR), Barcelona, 3-8 September 2000, Vol. 4, (2000) 775-778 
7. K. Obermayer, T.J. Sejnowski (eds.): Self-Organizing Map Formation - Foundations of Neural Computation. The MIT Press, Cambridge Ma. (2001)

8. R. Rebmann, G. Krell, B. Michaelis: Reduction of Compression Artefacts Caused by JPEG Compression. Proceedings of the VIIP2002 (Sep. 9-12, 2002), ACTA Press, Malaga (2002) 271-275

9. C. Amerijckx and Ph. Thissen: Image Compression by Self-Organized Kohonen Map. IEEE Transactions on Neural Networks, Vol. 9, No. 3, May 1998 (503-507)

10. U. Seiffert, L.C. Jain (eds.): Self-Organizing Neural Networks - Recent Advances and Applications. Springer, Heidelberg (2001)

11. H.R. Tizhoosh, B. Michaelis: Image Enhancement Based on Fuzzy Aggregation techniques. IMTC/99, Proceedings of the 16th IEEE converence (1999) 1813-1817 